\title{
Universality of random graphs and rainbow embedding
}

\author{
Asaf Ferber \\ Rajko Nenadov \\ Ueli Peter*
}

November 9, 2018

\begin{abstract}
In this paper we show how to use simple partitioning lemmas in order to embed spanning graphs in a typical member of $\mathcal{G}(n, p)$. Let the maximum density of a graph $H$ be the maximum average degree of all the subgraphs of $H$. First, we show that for $p=\omega\left(\Delta^{12} n^{-1 / 2 d} \log ^{3} n\right)$, a graph $G \sim \mathcal{G}(n, p)$ w.h.p. contains copies of all spanning graphs $H$ with maximum degree at most $\Delta$ and maximum density at most $d$. For $d<\Delta / 2$, this improves a result of Dellamonica, Kohayakawa, Rödl and Rucińcki. Next, we show that if we additionally restrict the spanning graphs to have girth at least 7 then the random graph contains w.h.p. all such graphs for $p=\omega\left(\Delta^{12} n^{-1 / d} \log ^{3} n\right)$. In particular, if $p=\omega\left(\Delta^{12} n^{-1 / 2} \log ^{3} n\right)$, the random graph therefore contains w.h.p. every spanning tree with maximum degree bounded by $\Delta$. This improves a result of Johannsen, Krivelevich and Samotij.

Finally, in the same spirit, we show that for any spanning graph $H$ with constant maximum degree, and for suitable $p$, if we randomly color the edges of a graph $G \sim \mathcal{G}(n, p)$ with $(1+o(1))|E(H)|$ colors, then w.h.p. there exists a rainbow copy of $H$ in $G$ (that is, a copy of $H$ with all edges colored with distinct colors).
\end{abstract}

\section{Introduction}

A graph $G$ is universal for a family of graphs $\mathcal{H}$ (we write $G$ is $\mathcal{H}$-universal), if $G$ contains a copy of every graph $H \in \mathcal{H}$. The construction (explicit and/or randomized) of sparse universal graphs for various families has received a considerable amount of attention (see [1, 2, 3, 4, 6, 7, 9, 10, 11, 12, 13, 15, 19, 21]).

In particular, the probability space $\mathcal{G}(n, p)$ of all graphs on $n$ vertices, in which each pair of vertices forms an edge with probability $p$ independently at random, has been considered in many papers. The problem of finding for which values of $p$ a typical member of $\mathcal{G}(n, p)$ is $\mathcal{H}$-universal for various families of graphs is fundamental in the theory of random graphs.

*Institute of Theoretical Computer Science ETH, 8092 Zürich, Switzerland. Emails: asaf.ferber@inf.ethz.ch, rnenadov@inf.ethz.ch and upeter@inf.ethz.ch 
Let $\mathcal{H}(n, \Delta, d)$ be the family of all graphs on $n$ vertices with maximum degree at most $\Delta$ and with maximum density at most $d$, where the maximum density of a graph $G$ (denoted by $d(G)$ ) is defined as

$$
d(G)=\max \left\{\frac{2|E(H)|}{|V(H)|}: H \subseteq G\right\} .
$$

Dellamonica, Kohayakawa, Rödl and Rucińcki proved in [15] that for maximum degree $\Delta \geq 3$ and an edge probability $p=\omega\left(n^{-1 / \Delta} \log ^{1 / \Delta} n\right)$, a typical member of $\mathcal{G}(n, p)$ is $\mathcal{H}(n, \Delta, \Delta)$ universal. Recently, Kim and Lee [21] obtained similar bounds for $\Delta=2$. In the following theorem we show that if $d<\Delta / 2$, then the bound in [15] can be further improved.

Theorem 1.1 Let $n$ be a positive integer, and let $\Delta=\Delta(n)>1$ and $d=d(n) \geq 2$ be integers. Then for $p=\omega\left(\Delta^{12} n^{-1 / \min \{2 d, \Delta\}} \log ^{3} n\right)$, a graph $G \sim \mathcal{G}(n, p)$ is w.h.p. $\mathcal{H}(n, \Delta, d)$-universal.

To prove this theorem, it will be sufficient to prove that it holds for $p=\omega\left(\Delta^{12} n^{-1 /(2 d)} \log ^{3} n\right)$ as it follows from [15] for the other minimum.

Next, let $\mathcal{H}(n, \Delta, d, g) \subseteq \mathcal{H}(n, \Delta, d)$ denote the family of graphs which additionally have girth at least $g$ (the girth of a graph is the length of its shortest cycle). In our second main result we further restrict ourselves to graphs with girth at least 7, where we obtain better bounds for $p$.

Theorem 1.2 Let $n$ be a positive integer, and let $d=d(n)$ and $\Delta=\Delta(n)>1$ be integers. Then for $p=\omega\left(\Delta^{12} n^{-1 / d} \log ^{3} n\right)$, a graph $G \sim \mathcal{G}(n, p)$ is w.h.p. $\mathcal{H}(n, \Delta, d, 7)$-universal.

Another example of a family of graphs which has attracted the attention of various researchers is the family of bounded degree trees. Let $\mathcal{T}(n, \Delta)$ be the family of all forests on $n$ vertices with maximum degree bounded by $\Delta$. Alon, Krivelevich and Sudakov showed in [6] that for fixed $\Delta>0$ and $0<\varepsilon<1$, there exists a constant $c=c(\Delta, \varepsilon)$ such that a typical member of $\mathcal{G}(n, c / n)$ is $\mathcal{T}((1-\varepsilon) n, \Delta)$-universal. The constant $c$ in this result was further improved in [8]. Later on, Balogh, Csaba and Samotij showed in [9] that $\mathcal{G}(n, c / n)$ is w.h.p. (with high probability) $\mathcal{T}((1-\varepsilon) n, \Delta)$-universal even if an adversary is allowed to delete at most (roughly) half of the edges touching any vertex. Note that universality for spanning trees can not be true for $p=c / n$, as at such a low density the random graph is w.h.p. disconnected. As it turns out, results for spanning subgraphs are much harder to obtain. In the case of the family of spanning trees $\mathcal{T}(n, \Delta)$, the best bound known for $\mathcal{G}(n, p)$ to be $\mathcal{T}(n, \Delta)$-universal is $p=\omega\left(\Delta n^{-1 / 3} \log ^{2} n\right)$, due to Johannsen, Krivelevich and Samotij [19]. The following immediate corollary of Theorem 1.2 improves this bound to $p=\omega\left(\Delta^{12} n^{-1 / 2} \log ^{3} n\right)$.

Corollary 1.3 Let $n$ be a positive integer, and let $\Delta=\Delta(n)>1$ be an integer. Then for $p=\omega\left(\Delta^{12} n^{-1 / 2} \log ^{3} n\right)$, a graph $G \sim \mathcal{G}(n, p)$ is w.h.p. $\mathcal{T}(n, \Delta)$-universal.

The proofs of Theorems 1.1 and 1.2 use simple partitioning lemmas for graphs and an embedding technique based on matchings, developed by Alon and Füredi in [5] and by Ruciński in 
[22]. Using similar technique, we also managed to obtain a general embedding result in a model of random graphs where each edge is being colored uniformly at random in one color from a given set of colors. This leads us to the second part of our paper.

Let $G \sim \mathcal{G}(n, p)$ and assume that each edge of $G$ is colored uniformly at random with one of the colors from the set $[c]:=\{1, \ldots, c\}$. This model is referred to as $\mathcal{G}_{c}(n, p)$. For a given graph $H$ we say that a typical member of $G \sim \mathcal{G}_{c}(n, p)$ contains a rainbow copy of $H$, if $G$ contains as a subgraph a copy of $H$ with all the edges colored in distinct colors. In [17], Frieze and Loh showed that for $p \geq(1+\varepsilon) \log n / n$ and $c=n+o(n)$, a typical member of $\mathcal{G}_{c}(n, p)$ contains a rainbow Hamilton cycle. Note that their result is asymptotically optimal in both $p$ and the number of colors $c$. In the following theorem we provide bounds on the edge probability $p$ (do not believed to be optimal), for which given any graph $H$ on $n$ vertices with $\Delta(H)=O(1)$, one can find a rainbow copy of $H$ in a typical member of $\mathcal{G}_{c}(n, p)$, provided $c=(1+o(1))|E(H)|(c$ is asymptotically optimal).

Theorem 1.4 Let $\alpha>0$, let $\Delta$ and $d$ be integers, let $n$ be a sufficiently large integer and let $H \in \mathcal{H}(n, \Delta, d)$. Then $G \sim \mathcal{G}_{c}(n, p)$ w.h.p. contains a rainbow copy of $H$, provided that $p \geq n^{-1 / d} \log ^{5 / d} n$ and $c=(1+\alpha)|E(H)|$.

We remark that all of our proofs might be easily improved in terms of $\log n$ and $\Delta$ factors. Since we believe that our bounds are far from being optimal, we did no effort in optimizing those factors.

Notation. Our graph-theoretic notation is standard and follows that of [23]. For a graph $G$, let $V=V(G)$ and $E=E(G)$ denote its sets of vertices and edges, respectively. For subsets $U, W \subseteq V$, and for a vertex $v \in V$, we denote by $E_{G}(U)$ all the edges of $G$ with both endpoints in $U$, by $E_{G}(U, W)$ all the edges of $G$ with one endpoint in $U$ and one endpoint in $W$ and by $E_{G}(v, U)$ all the edges with one endpoint being $v$ and one endpoint in $U$. We write $N_{G}(v)$ for the neighborhood of $v$ in $G$ and $\operatorname{deg}_{G}(v)$ for its degree. Moreover, we write $N_{G}(U)$ for the neighborhood of a set $U \subseteq V$. For any positive integer $k$ and every vertex $V$ we denote the following set as $k$-neighborhood of $v$ :

$$
\{v \in V \mid \text { the distance between } u \text { and } v \text { is at most } k\}
$$

We say that a set $S \subseteq V$ is $k$-independent if and only if (in $G$ ) the distance between any two vertices of $S$ is at least $k+1$.

Given a graph $G$ and a positive constant $d>0$ we denote by $D_{d}(G)$ the set of all vertices of $G$ with degree exactly $d$, by $D_{\leq d}(G)$ the set of all vertices of degree at most $d$ and in a similar way we define $D_{<d}(G), D_{>d}(G)$ and $D_{\geq d}(G)$. When it is clear to which graph $G$ we refer, we just denote it by $D_{d}, D_{\leq d}$ etc.

Given two graphs $H$ and $G$, a bijection $f$ from $V(H)$ to $V(G)$ is called an embedding of $H$ to $G$ if it maps each edge of $H$ to an edge of $G$. In case that one assigns colors to the edges of $G$, an embedding $f$ of $H$ to $G$ is called a rainbow embedding if in addition it maps the edges of $H$ into edges with distinct colors in $G$. 
Throughout the paper, wherever we use $\log n$ we refer to the natural logarithm.

\section{Preliminaries}

\subsection{Probabilistic Tools}

We will need to employ bounds on large deviations of random variables. We will mostly use the following well-known bound on the lower and the upper tails of the binomial distribution due to Chernoff (see [18]).

Lemma 2.1 If $X \sim \operatorname{Bin}(n, p)$, then

- $\operatorname{Pr}(X<(1-a) n p)<e^{-a^{2} n p / 2}$ for every $a>0$;

- $\operatorname{Pr}(X>(1+a) n p)<e^{-a^{2} n p / 3}$ for every $0<a<3 / 2$.

The proof of the following slightly more general bounds follows directly from the Chernoff bound and is left as an exercise for the reader (see for example Problem 1.7 in [16]).

Lemma 2.2 Let $p, q \in[0,1]$ and let $X_{1}, \ldots, X_{n} \in\{0,1\}$ be $n$ indicator variables and $X:=$ $\sum_{i=1}^{n} X_{i}$. If for each $1 \leq i \leq n$

$$
\mathbb{E}\left[X_{i} \mid X_{1}, \ldots, X_{i-1}\right] \geq p \quad \text { and } \quad \mathbb{E}\left[X_{i} \mid X_{1}, \ldots, X_{i-1}\right] \leq q,
$$

then it holds for every $0<\alpha<1$ that

$$
\operatorname{Pr}[X \geq(1+\alpha) n q] \leq e^{-\alpha^{2} n q / 3} \quad \text { and } \quad \operatorname{Pr}[X \leq(1-\alpha) n p] \leq e^{-\alpha^{2} n p / 2} .
$$

\subsection{Graph-Theoretic Facts}

In this section we mention a few facts about graphs which are used extensively throughout the paper.

The first two lemmas consider the existence of $k$-independent sets in a graph.

Lemma 2.3 Let $G$ be a graph on $n$ vertices with maximum degree $\Delta \geq 2$ and let $S \subseteq V(G)$ be such that the maximum degree of all vertices in $S$ is at most $d$ (where $d \geq 1$ ). Then, $S$ contains a set $U \subseteq S$ of size at least $\frac{|S|}{d \Delta^{k}}$ which is k-independent in $G$.

Proof Build $U$ greedily as follows: start with $L:=S$ and $U:=\emptyset$. In each step add an arbitrary vertex $v \in L$ to $U$ and delete the $k$-neighborhood of $v$ (including $v$ itself) from $L$. Since after each addition of a vertex to $U$ we delete at most 


$$
1+d+d(\Delta-1)+\ldots+d(\Delta-1)^{k-1} \leq d \Delta^{k}
$$

vertices from $L$, we obtain the required.

Lemma 2.4 Let $G$ be a graph on $n$ vertices with maximum degree $\Delta \geq 2$ and let $d$ be an integer such that $d n \geq 2|E(G)|$. Then, for any integer $k, G$ contains a $k$-independent set $U \subseteq D_{\leq d}(G)$ of size $|U| \geq \frac{n}{(d+1) d \Delta^{k}}$.

Proof First, we claim that $\left|D_{\leq d}(G)\right| \geq \frac{n}{d+1}$. Indeed, let $G$ be a graph which satisfies the conditions of the lemma for some $\Delta$. Using the fact that $\left|D_{>d}\right|=n-\left|D_{\leq d}\right|$, we obtain that

$$
d n \geq \sum_{v \in V(G)} \operatorname{deg}_{G}(v) \geq 0 \cdot\left|D_{\leq d}\right|+(d+1) \cdot\left(n-\left|D_{\leq d}\right|\right) .
$$

Therefore, we conclude that $\left|D_{\leq d}\right| \geq \frac{n}{d+1}$.

Applying Lemma 2.3 we conclude that there exists a $k$-independent set $U \subseteq D_{\leq d}$ in $G$ of size at least

$$
|U| \geq \frac{\left|D_{\leq d}\right|}{d \Delta^{k}} \geq \frac{n}{(d+1) d \Delta^{k}},
$$

as required.

A graph $G$ is called $d$-degenerate if every subgraph $G^{\prime} \subseteq G$ contains a vertex of induced degree at most $d$. A moment's thought reveals that every graph $H \in \mathcal{H}(n, \Delta, d)$ is $d$-degenerate (but not vice versa). The following observation follows directly from the definition of $d$-degenerate graphs.

Observation 2.5 Let $n, \Delta$ and $d$ be positive integers and let $H$ be a d-degenerate graph on $n$ vertices. Then there exists an ordering $\left(v_{1}, \ldots, v_{n}\right)$ of the vertices of $H$ such that

$$
\left|N\left(v_{i}\right) \cap\left\{v_{1}, \ldots, v_{i-1}\right\}\right| \leq d
$$

for every $2 \leq i \leq n$.

\section{Partitioning Lemmas}

In this section we prove some lemmas about partitioning graphs from $\mathcal{H}(n, \Delta, d)$ and $\mathcal{T}(n, \Delta)$. Before that, we define a class of graphs which can be partitioned in a "nice" way, and then we show that $\mathcal{H}(n, \Delta, d)$ and $\mathcal{T}(n, \Delta)$ belong to this class for suitably chosen parameters.

Definition 3.1 Let $n, d$ and $t$ be positive integers and let $\varepsilon$ be a positive number. The family of graphs $\mathcal{F}(n, t, \varepsilon, d)$ consists of all graphs $H$ on $n$ vertices for which the following holds. There exists a partition $V(H)=W_{0} \cup \ldots \cup W_{t}$ such that: 
(i) $\left|W_{t}\right|=\lfloor\varepsilon n\rfloor$,

(ii) $W_{0}=N\left(W_{t}\right)$,

(iii) $W_{t}$ is 3-independent,

(iv) $W_{i}$ is 2 -independent for every $1 \leq i \leq t-1$, and

(v) for every $1 \leq i \leq t$ and for every $w \in W_{i}$, w has at most $d$ neighbors in $W_{0} \cup \ldots \cup W_{i-1}$.

Now, we show that $\mathcal{H}(n, \Delta, d) \subseteq \mathcal{F}\left(n, 4 \Delta^{6} \log n+1, \varepsilon, 2 d\right)$.

Lemma 3.2 Let $n$ be a positive integer, let $\Delta=\Delta(n) \geq 2$ and $d=d(n) \geq 2$ be integers and let $\varepsilon_{0}=1 /\left(4 \Delta^{6}\right)$. Then for every $\varepsilon \leq \varepsilon_{0}$ we have

$$
\mathcal{H}(n, \Delta, d) \subseteq \mathcal{F}\left(n, 4 \Delta^{6} \log n+1, \varepsilon, 2 d\right) .
$$

Proof Let $H \in \mathcal{H}(n, \Delta, d)$ and $t=4 \Delta^{6} \log n+1$. We show that $H \in \mathcal{F}(n, t, \varepsilon, 2 d)$, for every $\varepsilon \leq \varepsilon_{0}$.

Using Lemma 2.4, one can find a 4-independent set $U \subseteq D_{\leq d}(H)$ of size

$$
|U| \geq \frac{n}{(d+1) d \Delta^{4}} \geq \varepsilon_{0} n .
$$

Let $W_{t} \subseteq U$ be an arbitrary subset of size $\lfloor\varepsilon n\rfloor$, and set $W_{0}=N_{H}\left(W_{t}\right)$ and $H_{t-1}:=H \backslash\left(W_{0} \cup W_{t}\right)$. We further partition $H_{i}$, for $i=t-1, \ldots, 1$, as follows:

- If $V\left(H_{i}\right)=\emptyset$ then set $W_{i}:=\emptyset$ and $V\left(H_{i-1}\right):=\emptyset$.

- Otherwise, $H_{i} \in \mathcal{H}\left(\left|H_{i}\right|, \Delta, d\right)$ and thus by Lemma 2.4 there exists a 2 -independent set $U \subseteq D_{\leq d}\left(H_{i}\right)$ of size $|U| \geq \frac{\left|H_{i}\right|}{(d+1) d \cdot \Delta^{2}} \geq \frac{\left|H_{i}\right|}{2 \Delta^{4}} \geq \varepsilon_{0}\left|H_{i}\right|$. Set $W_{i}:=U$ and $H_{i-1}:=H_{i} \backslash W_{i}$.

Using the fact that $\log (1-x) \leq-x$ for every $0<x<1$, we have that

$$
t=4 \Delta^{6} \log n+1=\log n / \varepsilon_{0}+1 \geq-\log n / \log \left(1-\varepsilon_{0}\right)+1=-\log _{1-\varepsilon_{0}} n+1 .
$$

Since for each $i$ we have that $\left|V\left(H_{i}\right)\right| \leq\left(1-\varepsilon_{0}\right)\left|V\left(H_{i+1}\right)\right|$, and since $t \geq-\log _{1-\varepsilon_{0}} n+1$, it follows that $\left|V\left(H_{1}\right)\right| \leq 1$.

Now, let $V(H)=W_{0} \cup \ldots \cup W_{t}$ be the obtained partition and note that each vertex $w \in W_{i}$ has at most $d$ neighbors in $W_{1} \cup \ldots \cup W_{i-1}$ for $2 \leq i<t$ (it follows immediately from the construction). Since all the properties $(i)-(i v)$ of Definition 3.1 follow easily from the construction, it thus remains to show that Property $(v)$ holds. That is, we need to show that every vertex in $w \in W_{1} \cup \ldots \cup W_{t-1}$ has at most $d$ neighbors in $W_{0}$, and then we conclude that every vertex in $W_{1} \cup \ldots \cup W_{t}$ sends at most $2 d$ "back-edges". For this aim, note first that every vertex in 
$W_{t}$ has at most $d$ neighbors in $W_{0}$, and that $W_{0}=N_{H}\left(W_{t}\right)$. Therefore, if there exists a vertex $w \in W_{1} \cup \ldots \cup W_{t-1}$ with at least $d+1$ neighbors in $W_{0}$, then there must exist at least two vertices $x, y \in W_{t}$ such that $N_{H}(x) \cap N_{H}(w) \neq \emptyset$ and $N_{H}(y) \cap N_{H}(w) \neq \emptyset$. Therefore, one can find a path of length four between $x$ and $y$, which clearly contradicts the assumption that $W_{t}$ is 4-independent. This completes the proof.

Next, we show that $\mathcal{H}(n, \Delta, d, 7) \subseteq \mathcal{F}\left(n, 16 d^{2} \Delta^{2} \log n+1, \varepsilon, d\right)$.

Lemma 3.3 Let $n$ be a positive integer, let $\Delta=\Delta(n)$ and $d=d(n) \geq 2$ be integers, and let $\varepsilon_{0}=1 /\left(2 d^{2} \Delta^{6}\right)$. Then for every $\varepsilon \leq \varepsilon_{0}$ we have

$$
\mathcal{H}(n, \Delta, d, 7) \subseteq \mathcal{F}\left(n, 16 d^{2} \Delta^{2} \log n+1, \varepsilon, d\right) .
$$

Proof Let $\gamma=\frac{1}{8(d+1)(d-1) \Delta^{2}} \leq \frac{1}{d^{2}}$ and observe that for $d \geq 2$

$$
\frac{1-(d+1)(d-1) \Delta^{2} \gamma}{(d+1)(d-1) \Delta^{2}}>\gamma \quad \text { and } \quad \frac{d^{2}\left(1-(d+1)(d-1) \Delta^{2} \gamma\right)}{d+1}>1
$$

Let $H \in \mathcal{H}(n, \Delta, d, 7)$ and $t=16 d^{2} \Delta^{2} \log n+1$. We show that $H \in \mathcal{F}(n, t, \varepsilon, d)$, for every $\varepsilon \leq \varepsilon_{0}$.

Using Lemma 2.4, we find a 6-independent set $U \subseteq D_{\leq d}(H)$ of size

$$
|U| \geq \frac{n}{d(d+1) \Delta^{6}} \geq \varepsilon_{0} n
$$

For a fixed $\varepsilon \leq \varepsilon_{0}$, let $W_{t} \subseteq U$ be an arbitrary subset of size $\lfloor\varepsilon n\rfloor$, and set $W_{0}=N_{H}\left(W_{t}\right)$, $X=N_{H}\left(W_{0}\right)$, and $H_{t-1}:=H \backslash\left(W_{0} \cup W_{t}\right)$.

For $i=t-1, \ldots, 1$, we iteratively find subsets of vertices $W_{i} \subseteq V\left(H_{i}\right)$ (and set $\left.H_{i-1}:=H_{i} \backslash W_{i}\right)$, in such a way that at the end of the process the obtained partition $V(H)=W_{0} \cup \ldots \cup W_{t}$ satisfies Properties $(i)-(v)$ of Definition 3.1 .

If $V\left(H_{i}\right)=\emptyset$ then set $W_{i}:=\emptyset$ and $V\left(H_{i-1}\right):=\emptyset$. Otherwise, construct $W_{i}$ as follows:

(1) If there exists a 2-independent set $U \subseteq D_{\leq d-1}\left(H_{i}\right)$ of size $U \geq \gamma\left|V\left(H_{i}\right)\right|$, then set $W_{i}:=U$.

(2) Otherwise, pick a 2-independent set $W_{i} \subseteq D_{\leq d}\left(H_{i}\right) \backslash X$ of size $\left|W_{i}\right| \geq \gamma\left|V\left(H_{i}\right)\right|$.

Observe that a vertex can have at most one neighbor in $W_{0}$. Otherwise, we would either have that $W_{t}$ is not 6-independent or that there exists a cycle of length 4 in $H$, both yielding a contradiction. Therefore, by the definition of $W_{i}$, we ensure that Property $(v)$ of Definition 3.1 is satisfied. We now claim that whenever (1) fails, there exists a 2-independent set $U \subseteq D_{\leq d}\left(H_{i}\right)$ of size $|U| \geq \gamma n_{i}$ (where $n_{i}=\left|V\left(H_{i}\right)\right|$ ) such that $U \cap X=\emptyset$ as required in (2). We remark that we always consider the graph $H_{i}$ when we write $D_{d}, D_{\leq d}$ or $D_{\geq d}$ in the following calculations. 
To prove our claim, suppose that there is no 2-independent set $U \subseteq D_{\leq d-1}\left(H_{i}\right)$ of size at least $\gamma n_{i}$. First, note that by Lemma 2.3 we have

$$
\left|D_{\leq d-1}\right| \leq(d-1) \Delta^{2} \gamma n_{i}
$$

Second, since $H_{i} \in \mathcal{H}\left(n_{i}, \Delta, d, 7\right)$, it follows that

$$
d n_{i} \geq \sum_{v \in V\left(H_{i}\right)} \operatorname{deg}_{H_{i}}(v) \geq 0 \cdot\left|D_{\leq d-1}\right|+\left(\left|D_{\leq d}\right|-\left|D_{\leq d-1}\right|\right) \cdot d+\left(n_{i}-\left|D_{\leq d}\right|\right) \cdot(d+1)
$$

and therefore

$$
\left|D_{\leq d}\right| \geq n_{i}-\left|D_{\leq d-1}\right| \cdot d
$$

Using the bound on $\left|D_{\leq d-1}\right|$, we get that

$$
\left|D_{d}\right|=\left|D_{\leq d}\right|-\left|D_{\leq d-1}\right| \geq n_{i}-(d+1)\left|D_{\leq d-1}\right| \geq n_{i} \cdot\left(1-(d+1)(d-1) \Delta^{2} \gamma\right) .
$$

Next, note that if $\left|X \cap D_{d}\right| \leq d\left|D_{d}\right| /(d+1)$, then by Lemma 2.3 there exists a 2-independent set $W_{i} \subseteq D_{d} \backslash X$ of size at least

$$
\left|W_{i}\right| \geq \frac{\left|D_{d} \backslash X\right|}{d \Delta^{2}} \stackrel{(2)}{\geq} \frac{1-(d+1)(d-1) \Delta^{2} \gamma}{d(d+1) \Delta^{2}} n_{i} \stackrel{(1)}{\geq} \gamma n_{i}
$$

as required. Therefore, assume that $\left|X \cap D_{d}\right|>d\left|D_{d}\right| /(d+1)$. Observe that $X$ is a 2-independent set in $H_{i}$, as every vertex in $X$ is a neighbor of a vertex in $W_{0}=N_{H}\left(W_{t}\right), W_{t}$ is 6-independent and there are no cycles of length at most 6 in $H$. It thus follows that $N_{H_{i}}(X) \cap X=\emptyset$ and every vertex in $N_{H_{i}}(X)$ has exactly one neighbor in $X$. Therefore,

$$
\left|N_{H_{i}}(X)\right| \geq d\left|X \cap D_{d}\right|>d^{2}\left|D_{d}\right| /(d+1)
$$

and it follows from (2) that

$$
\left|N_{H_{i}}(X)\right|>\frac{d^{2}}{d+1} \cdot\left(1-(d+1)(d-1) \Delta^{2} \gamma\right) n_{i} \stackrel{\mathbb{1} 1}{>} n_{i}
$$

which is not possible. Hence, one can always find a 2-independent set $W_{i} \subseteq V\left(H_{i}\right)$ of size at least $\gamma n_{i}$ as required.

Using the fact that $\log (1-x) \leq-x$ for every $0<x<1$, we have that

$$
t+1=16 d^{2} \Delta^{2} \log n+1 \geq \log n / \gamma+1 \geq-\log n / \log (1-\gamma)+1=-\log _{1-\gamma} n+1
$$

Since for each $i$ we have that $\left|V\left(H_{i}\right)\right| \leq(1-\gamma)\left|V\left(H_{i+1}\right)\right|$, and since $t \geq-\log _{1-\gamma} n+1$, it follows that $\left|V\left(H_{1}\right)\right| \leq 1$. Finally, let $V(H)=W_{0} \cup \ldots \cup W_{t}$ be the obtained partition. It follows immediately from the construction that Properties $(i)-(v)$ of Definition 3.1 hold. This completes the proof. 


\section{Proof of Theorem 1.1 and Theorem 1.2}

In this section we prove Theorem 1.1 and Theorem 1.2. These theorems follow easily from the following theorem and Lemma 3.3 and 3.2 .

Theorem 4.1 Let $n$ and $t$ be positive integers, let $d=d(n) \geq 2$ be an integer, and let $\varepsilon<\frac{1}{2 d}$. Then, a graph $G \sim \mathcal{G}(n, p)$ is w.h.p. $\mathcal{F}(n, t, \varepsilon, d)$-universal, provided that $p=\omega\left(\varepsilon^{-1} t n^{-1 / d} \log ^{2} n\right)$.

In order to prove Theorem 4.1, we use a similar embedding algorithm as the one presented in [21] (and previously in [15]). Let $d$ be a positive integer and $\varepsilon$ be a positive constant. Our goal is to show that, whenever a graph $G$ is "good" with respect to some properties, then $G$ is $\mathcal{F}(n, t, \varepsilon, d)$-universal.

Before we state formally what a "good" graph is, we define the following auxiliary bipartite graph. For a graph $G$, an integer $k$, a subset $U \subseteq V(G)$ and a collection $\mathcal{L}$ of pairwise disjoint $k$-subsets of $V(G) \backslash U$, define the bipartite graph $\mathcal{B}(\mathcal{L}, U)$ as follows: the parts are $\mathcal{L}$ and $U$, and two elements $L \in \mathcal{L}$ and $u \in U$ are adjacent if and only if $L \subseteq N_{G}(u)$. Now we can define the notion of an $(n, t, \varepsilon, d)$-good graph $G$.

Definition 4.2 A graph $G$ on $n$ vertices is called $(n, t, \varepsilon, d)$-good if there exists a partition $V(G)=V_{0} \cup V_{1} \cup \cdots \cup V_{t}$ with

$$
\left|V_{i}\right|=\frac{\varepsilon n}{16 t} \quad \text { for } 1 \leq i \leq t \quad \text { and } \quad\left|V_{0}\right|=\left(1-\frac{\varepsilon}{16}\right) n,
$$

such that for $p \geq \varepsilon^{-1} t n^{-1 / d} \log ^{2} n$ the following properties hold.

(P1) There exists a set $\mathcal{K} \subset V_{0}$ of $\varepsilon n$ vertex-disjoint d-cliques such that for all $U \subset V(G) \backslash V(\mathcal{K})$ with $|U| \leq(p / 2)^{-d} / 2$, we have

$$
\mid\left\{K_{d} \in \mathcal{K} \mid V\left(K_{d}\right) \subset N_{G}(u) \text { for some } u \in U\right\}\left|\geq \frac{1}{2^{d+2}} p^{d}\right| U \mid \varepsilon n .
$$

(P2) Let $1 \leq k \leq d$, and $\mathcal{L}$ be a collection of pairwise disjoint $k$-subsets of $V(G)$.

If $|\mathcal{L}| \leq(p / 2)^{-k} / 2$, then for each $i=1, \ldots, t$ with $V_{i} \cap\left(\cup_{L \in \mathcal{L}} L\right)=\emptyset$, we have that

$$
\left|N_{\mathcal{B}\left(\mathcal{L}, V_{i}\right)}(\mathcal{L})\right| \geq(p / 2)^{k}|\mathcal{L}|\left|V_{i}\right| / 2 .
$$

If $|\mathcal{L}| \geq(p / 2)^{-k} \log ^{2(d-1)} n$, then for all $U$ with $|U| \geq(p / 2)^{-k} \log ^{2(d-1)} n$ and $U \cap\left(\cup_{L \in \mathcal{L}} L\right)=$ $\emptyset$, the graph $\mathcal{B}(\mathcal{L}, U)$ has at least one edge.

We first show that a random graph is typically good.

Lemma 4.3 Let $\varepsilon<\frac{1}{2 d}$ and let $n$ be a positive integer. Then, a graph $G \sim \mathcal{G}(n, p)$ is w.h.p. $(n, t, \varepsilon, d)$-good, provided that $p=\omega\left(\varepsilon^{-1} t n^{-1 / d} \log ^{2} n\right)$. 
Proof Let $\varepsilon \leq \frac{1}{2 d}$, let $p=\omega\left(\varepsilon^{-1} t n^{-1 / d} \log ^{2} n\right)$ and let $G \sim \mathcal{G}(n, p)$. Furthermore, let $q \geq p / 2$ be such that $1-p=(1-q)^{2}$, and note that one can expose $G \sim \mathcal{G}(n, p)$ as $G=G_{1} \cup G_{2}$, where $G_{1}$ and $G_{2}$ are two graphs sampled from $\mathcal{G}(n, q)$ independently (for more details we refer the reader to [18]). We use $G_{1}$ to find a family of vertex-disjoint $d$-cliques, and then $G_{2}$ to ensure the properties (P1) and (P2). For a simpler presentation, we assume from now on that $q$ is exactly $p / 2$.

First, expose the edges of $G_{1}$. Since

$$
q=\omega\left(n^{-2 / d}(\log n)^{1 /\left(\begin{array}{l}
d \\
2
\end{array}\right)}\right)
$$

it follows from [20] that $G_{1}$ contains w.h.p. $\lfloor n / d\rfloor$ disjoint $d$-cliques. Let $\mathcal{K}$ be a family of $\varepsilon n$ vertex-disjoint $d$-cliques. Next, fix an arbitrary partition $V(G)=V_{0} \cup \ldots \cup V_{t}$ as in Definition 4.2 , such that $V(\mathcal{K}) \subset V_{0}$. Finally, expose $G_{2}$. We now show that w.h.p. this partition satisfies Properties (P1) and (P2).

For $U \subseteq V(G) \backslash V(\mathcal{K})$ with $|U| \leq(p / 2)^{-d} / 2$, let

$$
X(U):=\mid\left\{K_{d} \in \mathcal{K} \mid K_{d} \subset N_{G_{2}}(u) \text { for some } u \in U\right\} \mid .
$$

Note that $X(U)$ is the sum of i.i.d. indicator random variables $X_{L}(L \in \mathcal{K})$, such that $X_{L}=1$ iff $L \subseteq N_{G_{2}}(u)$ for some $u \in U$. Since $|U| \leq(p / 2)^{-d} / 2$, we have that for each $L \in \mathcal{K}$,

$$
\operatorname{Pr}\left[X_{L}=0\right]=\left(1-(p / 2)^{d}\right)^{|U|} \leq 1-\frac{|U| p^{d}}{2^{d}}+\frac{|U|^{2} p^{2 d}}{2^{2 d}} \leq 1-\frac{|U| p^{d}}{2^{d}}(1-1 / 2)=1-\frac{|U| p^{d}}{2 \cdot 2^{d}}
$$

(For the first inequality we use the fact that $(1-a)^{b} \leq 1-a b+(a b)^{2}$ for any positive integer $b$ and $0<a<1)$.

Therefore, we have that $\operatorname{Pr}\left[X_{L}=1\right] \geq 2^{-d-1}|U| p^{d}$, which implies that

$$
\mathbb{E}[X(U)] \geq 2^{-d-1}|U| p^{d}|\mathcal{K}| \geq \frac{\log ^{2 d} n}{2^{d+1}}|U|
$$

Using Chernoff's bound we obtain that

$$
\operatorname{Pr}\left[X(U)<\frac{p^{d}}{2^{d+2}}|\mathcal{K}||U|\right] \leq 2 e^{-\frac{\log ^{2 d} n}{8 \cdot 2^{d+1}}|U|} \leq \frac{2}{n^{3|U|}} .
$$

(The last inequality holds since $d>1$ ).

We can therefore upper bound the probability that there exists a set $U$ that violates $(P 1)$ by the following union bound

$$
\sum_{\ell=1}^{n}\left(\begin{array}{l}
n \\
\ell
\end{array}\right) \frac{2}{n^{3 \ell}}=o(1)
$$

For property $(P 2)$ we first assume that $|\mathcal{L}| \leq(p / 2)^{-k} / 2$. Note that $X\left(\mathcal{L}, V_{i}\right):=\left|N_{\mathcal{B}\left(\mathcal{L}, V_{i}\right)}(\mathcal{L})\right|$ is the sum of i.i.d. indicator random variables $X_{v}$ (for $v \in V_{i}$ ), where $X_{v}=1$ iff $L \subset N_{G_{2}}(v$ ) 
for some $L \in \mathcal{L}$. Since $(p / 2)^{k}|\mathcal{L}| \leq 1 / 2$, using the fact that $(1-a)^{b} \leq 1-a b+(a b)^{2} / 2$ holds for every integer $b$ and any positive constant $a$ for which $a b<1$ (follows from the binomial formula), we observe that

$$
\mathbb{E}\left[X\left(\mathcal{L}, V_{i}\right)\right] \geq\left|V_{i}\right|\left(1-\left(1-(p / 2)^{k}\right)^{|\mathcal{L}|}\right) \geq(1-1 / 4)(p / 2)^{k}|\mathcal{L}|\left|V_{i}\right|
$$

Using Chernoff's bound we obtain that

$$
\operatorname{Pr}\left[X\left(\mathcal{L}, v_{i}\right)<(p / 2)^{k}|\mathcal{L}|\left|V_{i}\right| / 2\right] \leq \exp \left[-\mathbb{E}\left[X\left(\mathcal{L}, v_{i}\right)\right] / 36\right] \leq \frac{1}{n^{3 d|\mathcal{L}|}},
$$

where the last inequality follows as

$$
(p / 2)^{k}\left|V_{i}\right| \geq(p / 2)^{d} \cdot \frac{\varepsilon n}{16 t} \geq \frac{n \log ^{2 d} n}{2^{d+4} n}=\omega(d \log n) .
$$

Thus, the probability for having sets $\mathcal{L}$ and $V_{i}$ such that $\left|N_{\mathcal{B}\left(\mathcal{L}, V_{i}\right)}(\mathcal{L})\right|<(p / 2)^{k}|\mathcal{L}|\left|V_{i}\right| / 2$ can be bounded by

$$
\left.t \sum_{\ell=1}^{n}\left(\begin{array}{c}
n \\
k
\end{array}\right)\right) \frac{1}{n^{3 d \ell}}=o(1)
$$

Next, assume that $|\mathcal{L}| \geq(p / 2)^{-k} \log ^{2(d-1)} n$. Observe that each edge in $\mathcal{B}(\mathcal{L}, U)$ is present with probability $(p / 2)^{k}$, hence the probability that there are no edges is bounded by

$$
\left(1-(p / 2)^{k}\right)^{|\mathcal{L}||U|} \leq \exp \left[-(p / 2)^{k} \cdot|\mathcal{L}||U|\right]
$$

Furthermore, for $r, \ell \geq(p / 2)^{-k} \log ^{2(d-1)} n$, the number of collections of $k$-subsets $\mathcal{L}$ with $|\mathcal{L}|=\ell$ is at most $n^{k \ell}$, and the number of subsets $U$ with $|U|=r$ is at most $n^{r}$. We thus have that

$$
\operatorname{Pr}[\exists \mathcal{L}, U \text { with }|\mathcal{L}|=\ell,|U|=r \text { and } e(\mathcal{B}(\mathcal{L}, U))=0] \leq \exp \left[(k \ell+r) \log n-(p / 2)^{k} \ell r\right] .
$$

Note that

$$
(k \ell+r) \log n \leq k \cdot(\ell \log n+r \log n) \leq 2 k \cdot \frac{r \ell(p / 2)^{k}}{\log ^{2 d-3} n} \leq(p / 2)^{k} \ell r / 2
$$

for $n$ large enough, and hence,

$$
\exp \left[(k \ell+r) \log n-(p / 2)^{k} \ell r\right] \leq \exp \left[-(p / 2)^{k} \ell r / 2\right] \leq \exp \left[-(p / 2)^{-k} \log n / 2\right]=o(1) .
$$

We therefore conclude that the probability for the existence of such sets $\mathcal{L}$ and $U$ without an edge is $o(1)$.

Now we want to show that any $(n, t, \varepsilon, d)$-good graph is $\mathcal{F}(n, t, \varepsilon, d)$-universal. Let $G$ be a a $(n, t, \varepsilon, d)$-good graph with a partition $V(G)=V_{0} \cup \cdots \cup V_{t}$ and a clique-set $\mathcal{K}$. We construct an embedding $f: V(H) \rightarrow V(G)$ for a given graph $H \in \mathcal{F}(n, t, \varepsilon, d)$ as follows. 
Let $H=W_{0} \cup \cdots \cup W_{t}$ be the partition of $H$ that satisfies the conditions $(i)-(v)$ of Definition 4.2 . For every $v \in W_{t}$ let $L(v):=N_{G}(v) \cap W_{0}$ denote the neighborhood of $v$ in $W_{0}$. Note that since $W_{t}$ is 3-independent, we have that $L(u) \cap L(v)=\emptyset$ for $u \neq v$. In a first step we choose an arbitrary injective mapping $f_{0}: W_{0} \rightarrow V(\mathcal{K})$ such that for every $w \in W_{t}$ the vertices in $L(w)$ all map to vertices of the same clique in $\mathcal{K}$. Such a mapping exists as $\mathcal{K}$ consists of $\lfloor\varepsilon n\rfloor d$-cliques and there are exactly that many sets $L(w)$, each of which contains at most $d$ vertices. Moreover, such a mapping is valid as there can not be edges between $L(u)$ and $L(w)$ for $u \neq w$ (because $W_{t}$ is 3-independent).

For $i=1, \ldots, t$, we iteratively construct $f_{i}:\left(W_{0} \cup \cdots \cup W_{i}\right) \rightarrow\left(V_{0} \cup \cdots \cup V_{i}\right)$ from $f_{i-1}$ as follows. Let $V_{i}^{*}:=\left(V_{0} \cup \cdots \cup V_{i}\right) \backslash \operatorname{Img}\left(f_{i-1}\right)$. We want to embed $W_{i}$ to $V_{i}^{*}$. For $w \in W_{i}$ let $L_{i}(w):=f_{i-1}\left(N_{H}(w) \cap\left(\cup_{j=0}^{i-1} W_{j}\right)\right)$ and let $\mathcal{L}_{i}:=\left\{L_{i}(w) \mid w \in W_{i}\right\}$. Here it is crucial that $W_{i}$ is 2-independent and therefore $L_{i}(w) \cap L_{i}\left(w^{\prime}\right)=\emptyset$ for $w \neq w^{\prime} \in W_{i}$. Since a vertex $w \in W_{i}$ can be mapped only to the vertices in

$$
\left\{v \in V_{i}^{*} \mid L_{i}(w) \subseteq N_{G}(v)\right\},
$$

we can extend $f_{i-1}$ by a $\mathcal{L}_{i}$ matching in $B_{i}:=\mathcal{B}\left(\mathcal{L}_{i}, V_{i}^{*}\right)$ (recall that in $B_{i}$ the set $L_{i}(w) \in \mathcal{L}_{i}$ is connected to a vertex $v \in V_{i}^{*}$ if and only if $\left.L_{i}(w) \subseteq N_{G}(v)\right)$. More precisely, for a matching $\mathcal{M}$ which saturates $\mathcal{L}_{i}$ (an $\mathcal{L}_{i}$-matching), we define $f_{i}$ as follows: For $w \in W_{0} \cup \cdots \cup W_{i-1}$ let $f_{i}(w):=f_{i-1}(w)$, and for $w \in W_{i}$ let $f_{i}(w):=v$, where $v \in V_{i}^{*}$ is the unique vertex such that $\left\{L_{i}(w), v\right\} \in \mathcal{M}$.

As long as we find an $\mathcal{L}_{i}$-matching for $1 \leq i \leq t$ we clearly construct a valid embedding of $H$ into $G$. It remains to show that we can find the required matchings.

We first show that for every $1 \leq i \leq t-1$, the auxiliary graph $B_{i}$ contains an $\mathcal{L}_{i}$-matching.

Claim 4.4 For every $1 \leq i \leq t-1$, there exists an $\mathcal{L}_{i}$-matching in $B_{i}$.

Proof We show that Hall's condition for the existence of an $\mathcal{L}_{i}$-saturating matching is satisfied. First, we show that $\left|\mathcal{L}_{i}\right|=\left|W_{i}\right|<\left|V_{i}^{*}\right|-\frac{\varepsilon n}{16}$ for $1 \leq i \leq t-1$. We have

$$
\left|V_{i}^{*}\right|=\left|V_{0} \cup \cdots \cup V_{i}\right|-\left|W_{0} \cup \cdots \cup W_{i-1}\right|=\left|W_{i} \cup \cdots \cup W_{t}\right|-\left|V_{i+1} \cap \cdots \cup V_{t}\right|
$$

and therefore

$$
\left|V_{i}^{*}\right|-\left|W_{i}\right|=\left|W_{i+1} \cup \cdots \cup W_{t}\right|-\left|V_{i+1} \cap \cdots \cup V_{t}\right| \geq\left|W_{t}\right|-\frac{t-i}{16 t} \varepsilon n>\frac{15 \varepsilon n}{16} .
$$

Thus, we have that $\left|\mathcal{L}_{i}\right|=\left|W_{i}\right| \leq\left|V_{i}^{*}\right|-\frac{15 \varepsilon n}{16}<\left|V_{i}^{*}\right|-\frac{\varepsilon n}{16}$ and the claim therefore follows by Claim 4.5 below.

Claim 4.5 For all $U \subseteq \mathcal{L}_{i}$ that satisfy $|U| \leq\left|V_{i}^{*}\right|-\frac{\varepsilon n}{16}$, we have

$$
\left|N_{B_{i}}(U)\right| \geq|U| \text {. }
$$


Proof Let $U=U_{0} \cup \ldots \cup U_{d}$, where

$$
U_{j}:=\{L \in U|| L \mid=j\} .
$$

If $U_{0} \neq \emptyset$, then $N_{B_{i}}(U)=V_{i}^{*}$. Therefore, we may assume that $U_{0}=\emptyset$. Pick $k$ such that $\left|U_{k}\right| \geq|U| / d$. We show that the lemma holds for $n$ large enough by distinguish between the following three cases:

Case 1: $\left|U_{k}\right| \leq(p / 2)^{-k} / 2$. It follows by property $(P 2)$ that

$$
\left|N_{B_{i}}(U)\right| \geq\left|N_{B_{i}}\left(U_{k}\right)\right| \geq(p / 2)^{k}\left|U_{k}\right|\left|V_{i}\right| / 2 \geq \frac{\log ^{2 d} n}{2^{k+1} \cdot 16 d}|U| \geq|U| .
$$

Case 2: $(p / 2)^{-k} / 2 \leq\left|U_{k}\right| \leq(p / 2)^{-k} \log ^{2(d-1)} n$. We fix an arbitrary subset $U_{k}^{\prime} \subset U_{k}$ of size $\left|U_{k}^{\prime}\right|=(p / 2)^{-k} / 2$, and by the same argument as in Case 1 we get that

$$
\left|N_{B_{i}}(U)\right| \geq\left|N_{B_{i}}\left(U_{k}^{\prime}\right)\right| \geq(p / 2)^{k}\left|U_{k}^{\prime}\right|\left|V_{i}\right| / 2 \geq \frac{\log ^{2 d} n}{2^{k+1} \cdot 16}\left|U_{k}^{\prime}\right| \geq \frac{\log n}{2^{k+1} \cdot 16 d}|U| \geq|U| .
$$

Case 3: $\left|U_{k}\right| \geq(p / 2)^{-k} \log ^{2(d-1)} n$. In this case note that the induced subgraph $B_{i}\left[U_{k}, V_{i}^{*} \backslash\right.$ $\left.N_{B_{i}}\left(U_{k}\right)\right]$ has no edges. By property $(P 2)$ this yields that

$$
\left|V_{i}^{*} \backslash N_{B_{i}}\left(U_{k}\right)\right|<(p / 2)^{-k} \log ^{2(d-1)} n=o(\varepsilon n),
$$

which implies that $\left|N_{B_{i}}\left(U_{k}\right)\right| \geq\left|V_{i}^{*}\right|-o(\varepsilon n) \geq\left|V_{i}^{*}\right|-\frac{\varepsilon n}{16} \geq|U|$.

In the last lemma of this section we show that $B_{t}$ contains a perfect matching, thus we can complete the embedding of $H$.

Lemma 4.6 There exists a perfect matching in $B_{t}$.

Proof We check Hall's condition for every subset $U \subseteq \mathcal{L}_{t}$. For sets of cardinality $|U| \leq$ $\left|V_{t}^{*}\right|-\frac{\varepsilon n}{16}$, Hall's condition follows by Claim 4.5. Therefore, consider only subsets $U$ of cardinality $|U| \geq\left|V_{t}^{*}\right|-\frac{\varepsilon n}{16}$. Let $U \subseteq \mathcal{L}_{t}$ be such a subset. Note that by the definition of the partial embedding $f_{0}$, every set in $U$ is contained in one of the cliques in $\mathcal{K}$. Suppose first that $\left|V_{t}^{*} \backslash N_{B_{t}}(U)\right| \geq(p / 2)^{-d} / 2$. We fix a subset $Y \subset V_{t}^{*} \backslash N_{B_{t}}(U)$ of size exactly $(p / 2)^{-d} / 2$. It follows by property $(P 1)$ that at least $2^{-d-2} \cdot p^{d}|Y| \varepsilon n$ of the cliques in $\mathcal{K}$ are completely connected to some vertices in $Y$. We conclude that

$$
\left|N_{B_{t}}\left(V_{t}^{*} \backslash N_{B_{t}}(U)\right)\right| \geq\left|N_{B_{t}}(Y)\right| \geq 2^{-3}(p / 2)^{d}(p / 2)^{-d} \cdot \varepsilon n>\varepsilon n / 16,
$$

which is not possible since $|U|+\left|N_{B_{t}}\left(V_{t}^{*} \backslash N_{B_{t}}(U)\right)\right| \leq\left|V_{t}^{*}\right|$.

Therefore, we conclude that $\left|V_{t}^{*} \backslash N_{B_{t}}(U)\right| \leq(p / 2)^{-d} / 2$. Now, using Property $(P 1)$ similarly as above we obtain that

$$
\left|N_{B_{t}}\left(V_{t}^{*} \backslash N_{B_{t}}(U)\right)\right| \geq 2^{-d-3}(p / 2)^{d} \cdot \varepsilon n\left|V_{t}^{*} \backslash N_{B_{t}}(U)\right|>\left|V_{t}^{*} \backslash N_{B_{t}}(U)\right| .
$$

Finally, since

$$
\left|N_{B_{t}}(U)\right|+\left|V_{t}^{*} \backslash N_{B_{t}}(U)\right|=\left|V_{t}^{*}\right|=\left|W_{t}\right| \geq|U|+\left|N_{B_{t}}\left(V_{t}^{*} \backslash N_{B_{t}}(U)\right)\right|>|U|+\left|V_{t}^{*} \backslash N_{B_{t}}(U)\right|
$$

we get $\left|N_{B_{t}}(U)\right|>|U|$. 


\section{Proof of Theorem 1.4}

In this section we prove Theorem 1.4. Before starting the proof, it will be convenient to introduce the following notation. For any bipartite graph $G=(A \cup B, E)$ with $|A|=|B|=n$ and minimum degree $\delta(G) \geq k$, let $\mathcal{B}_{k-\text { out }}^{\ell}(G)$ denote the following set of bipartite graphs: each $D \in \mathcal{B}_{k-\text { out }}^{\ell}(G)$ has vertex set $V(D)=V(G)$ and edge set $E(D) \subseteq E$ such that each vertex in $A$ has degree exactly $k$. Note that we can sample an element from $\mathcal{B}_{k-\text { out }}^{\ell}(G)$ uniformly at random by choosing for each $v \in A$ uniformly at random $k$ edges from $E_{G}(v, B)$.

One of the main ingredients in the proof of Theorem 1.4 is the following simple lemma on the existence of perfect matchings in typical graphs from $\mathcal{B}_{k-\text { out }}^{\ell}(G)$.

Lemma 5.1 Let $\varepsilon>0$, let $n$ be a sufficiently large integer and let $k=\omega(\log n)$. Then for any bipartite graph $G=(A \cup B, E)$ with $|A|=|B|=n$ and $\delta(G) \geq \frac{n}{2}+\varepsilon n$, a graph $D$ chosen uniformly at random from $\mathcal{B}_{k-\text { out }}^{\ell}(G)$ w.h.p. contains a perfect matching.

Proof Let $D$ be a graph chosen uniformly at random from $\mathcal{B}_{k-\text { out }}^{\ell}(G)$. We show that w.h.p. all subsets $S \subset A$ and all subsets $S \subset B$ with $|S| \leq n / 2$ satisfy $|S| \leq\left|N_{D}(S)\right|$. It then follows from Hall's theorem (see [23] for more details) that $D$ has a perfect matching.

We first assume that $S \subset A$. Note that $|S|>\left|N_{D}(S)\right|$ implies that there exists a subset $S^{\prime} \subset B$ of size $\left|S^{\prime}\right|=|S|-1$ such that $\left|E_{D}\left(S, B \backslash S^{\prime}\right)\right|=0$. Note that in $G$, since $\left|S^{\prime}\right| \leq n / 2$, every vertex $v \in S$ has at least $\varepsilon n$ neighbors in $B \backslash S^{\prime}$. Therefore, when choosing the $i$-th of the $k$ edges incident to $v$ and conditioning on the event that no edge in $E_{G}\left(v, B \backslash S^{\prime}\right)$ has been selected so far, the probability to miss $B \backslash S^{\prime}$ is at most

$$
\frac{\operatorname{deg}_{G}(v)-\varepsilon n-i+1}{\operatorname{deg}_{G}(v)-i+1} \leq 1-\varepsilon .
$$

Thus,

$$
\begin{aligned}
\operatorname{Pr}\left[|S|>\left|N_{D}(S)\right|\right] & \leq \operatorname{Pr}\left[\exists S^{\prime} \subset B|| E_{D}\left(S, B \backslash S^{\prime}\right) \mid=0\right] \\
& \leq\left(\begin{array}{c}
n \\
|S|-1
\end{array}\right)(1-\varepsilon)^{|S| k} \leq e^{-\varepsilon|S| \cdot \omega(\log n)}
\end{aligned}
$$

and the probability that such a bad set exists is at most

$$
\sum_{s=1}^{n / 2}\left(\begin{array}{l}
n \\
s
\end{array}\right) e^{-\varepsilon s \cdot \omega(\log n)} \leq \sum_{s=1}^{n / 2} e^{-s \cdot \omega(\log n)}=o(1)
$$

Next, assume that $S \subset B$ and observe that in order to have $|S|>\left|N_{D}(S)\right|$, there must exist a set $S^{\prime} \subset A$ of size $|S|-1$ such that $\left|E_{D}\left(A \backslash S^{\prime}, S\right)\right|=0$. Note that $\left|E_{G}(A, S)\right| \geq|S| \cdot\left(\frac{n}{2}+\varepsilon n\right)$, $\left|E_{G}\left(S^{\prime}, S\right)\right| \leq|S| \cdot\left|S^{\prime}\right| \leq|S| \cdot \frac{n}{2}$ and therefore $\left|E_{G}\left(A \backslash S^{\prime}, S\right)\right| \geq|S| \varepsilon n$. Since every edge of $G$ 
appears in $D$ with probability at least $\frac{k}{n}$ (but not independently) and since this probability can only decrease if we know that another edge does not appear in $D$, it follows that

$$
\begin{aligned}
\operatorname{Pr}\left[|S|>\left|N_{D}(S)\right|\right] & \leq \operatorname{Pr}\left[\exists S^{\prime} \subset A|| E_{D}\left(A \backslash S^{\prime}, S\right) \mid=0\right] \\
& \leq\left(\begin{array}{c}
n \\
|S|-1
\end{array}\right)\left(1-\frac{\omega(\log n)}{n}\right)^{|S| \varepsilon n} \leq e^{-\varepsilon|S| \cdot \omega(\log n)}
\end{aligned}
$$

as in the previous case.

Now we are ready to prove Theorem 1.4 .

Proof Our proof is motivated by ideas of Cooper and Frieze [14. Note that containing a rainbow copy of some fixed graph $H$ is a monotone increasing property and we can therefore fix $p$ to exactly $n^{-1 / d} \log ^{5 / d} n$.

Let $\Delta$ and $d$ be positive integers, let $n$ be a sufficiently large integer and let $H \in \mathcal{H}(n, \Delta, d)$. Moreover, let $\bar{d}=\frac{2|E(H)|}{n}$ denote the average degree of $H$ (note that $\bar{d} \leq d$ and in fact can be much smaller than $d$ ) and let $\alpha>0$ be some arbitrarily small positive constant. First, we show how to partition $H$ in such a way that will later help us to find a rainbow copy of it in a typical member of $\mathcal{G}_{c}(n, p)$, where $c=(1+\alpha)|E(H)|$. For this aim we act as follows. If $H$ contains a set $W$ of $\left\lceil\frac{\alpha n}{5 \log ^{2} n}\right\rceil$ isolated vertices (that is, vertices of degree 0 in $H$ ), then partition $V(H)=\left\{w_{1}\right\} \cup \ldots \cup\left\{w_{t}\right\} \cup W$ in such a way that for each $i$, the vertex $w_{i}$ has at most $d$ neighbors in $\left\{w_{1}, \ldots, w_{i-1}\right\}$. Indeed, such a partition exists since $H^{\prime}:=H-W \in \mathcal{H}(n-|W|, \Delta, d)$, and therefore is $d$-degenerate, so one can apply Observation 2.5. Otherwise, let $x$ denote the number of vertices of degree larger than 0 and at most $\bar{d}$ in $H$. Since $H$ contains at most $\frac{\alpha n}{5 \log ^{2} n}$ isolated vertices, the following inequality holds:

$$
\bar{d} n=2|E(H)| \geq x+(\bar{d}+1)\left(n-\frac{\alpha n}{5 \log ^{2} n}-x\right) .
$$

Hence, using the fact that $n$ is sufficiently large, we conclude that $x \geq n /(2 \bar{d})$. Now, let $S$ be the set consisting of all these vertices. By applying Lemma 2.3 to $H$ and $S$ it follows that there exists a subset $T \subseteq S$, such that $T$ is 2 -independent and

$$
|T| \geq \frac{|S|}{\bar{d} \Delta^{2}} \geq \frac{n}{2 \bar{d}^{2} \Delta^{2}} \geq\left\lceil\frac{\alpha n}{5 \log ^{2} n}\right\rceil
$$

for sufficiently large $n$. Next, let $W \subseteq T$ be an arbitrary subset of size $\left\lceil\frac{\alpha n}{5 \log ^{2} n}\right\rceil$, and partition $V(H)=\left\{w_{1}\right\} \cup \ldots \cup\left\{w_{t}\right\} \cup W$ in such a way that for each $i, w_{i}$ has at most $d$ neighbors in $\left\{w_{1}, \ldots, w_{i-1}\right\}$.

All in all, we have a partition $V(H)=\left\{w_{1}\right\} \cup \ldots \cup\left\{w_{t}\right\} \cup W$ such that $|W|=\left\lceil\frac{\alpha n}{5 \log ^{2} n}\right\rceil$ and one of the following holds:

(1) all the vertices of $W$ are isolated in $H$, or 
(2) $W$ is 2-independent and consists of non-isolated vertices of degree at most $\bar{d}$.

Note that if (2) holds then

$$
|E(W, V \backslash W)| \leq \bar{d}|W|=\frac{2|E(H)|}{n} \cdot\left\lceil\frac{\alpha n}{5 \log ^{2} n}\right\rceil<\alpha|E(H)| /\left(2\left\lceil\log ^{2} n\right\rceil\right),
$$

for $n$ large enough.

Now we start to describe the procedure of finding a rainbow copy of $H$. Let $q \geq p / 2$ be such that $1-p=(1-q)^{2}$ and present $G \sim \mathcal{G}(n, p)$ as $G=G_{1} \cup G_{2}$, where $G_{1}$ and $G_{2}$ are two graphs sampled independently from $\mathcal{G}(n, q)$. We sample a member of $\mathcal{G}_{c}(n, p)$ by sampling a member of $\mathcal{G}(n, p)$ and randomly coloring exposed edges using $c$ colors.

We find a rainbow embedding of $H$ in $G \sim \mathcal{G}_{c}(n, p)$ in two phases. In Phase I, we find a rainbow embedding $f$ of $H\left[\left\{w_{1} \cup \ldots \cup w_{t}\right\}\right]$ with edges which are taken from $G_{1}$. If $W$ is as in (1) (that is, all the vertices in $W$ are isolated in $H$ ), then we are done. Otherwise, in Phase II we show that one can extend $f$ to a rainbow embedding of $H$ in $G$, using edges of $G_{2}$.

In what follows, we present the exact strategies of Phases I and II and prove that w.h.p. everything works out well.

Phase I: Throughout this phase we maintain a partial rainbow embedding $f$ of $H$ to $G_{1}$, a set of available colors $\mathcal{C}$ and a set of available vertices $V^{\prime}$. Initially, set $f=\emptyset, \mathcal{C}:=[c]$ and $V^{\prime}:=V(G)$. Additionally, we maintain for each vertex $v \in V(G)$ a set $U_{v} \subseteq V(G)$ such that $U(v) \cap V^{\prime}$ contains only unexposed potential neighbors of $v$ in $G_{1}$. Initially, $U_{v}=V(G) \backslash\{v\}$ for each $v \in V(G)$.

We inductively build the desired partial embedding $f$ as follows. In the first step, let $f\left(w_{1}\right):=v$ for an arbitrary vertex $v \in V^{\prime}$, and set $V^{\prime}:=V^{\prime} \backslash\{v\}$. Assume that we have already embedded $\left\{w_{1}, \ldots, w_{i-1}\right\}$ for some $2 \leq i \leq t$ and we wish to embed $w:=w_{i}$. Let $L\left(w_{i}\right)=f\left(N_{H}\left(w_{i}\right) \cap\right.$ $\left.\left\{w_{1}, \ldots, w_{i-1}\right\}\right)$ be the set of images of neighbors of $w_{i}$ which have already been embedded (recall that $\left.\left|L\left(w_{i}\right)\right| \leq d\right)$. Let $A_{w}=V^{\prime} \cap\left(\cap_{v \in L\left(w_{i}\right)} U_{v}\right)$ be the set of all available vertices which are still unexposed neighbors of all vertices in $L\left(w_{i}\right)$, and choose an arbitrary subset $S_{w} \subset A_{w}$ of size $s:=\left\lceil\alpha n /(4 \Delta \log n)^{2}\right\rceil$ (Claim 5.2 shows that throughout Phase I this is indeed possible; that is, $A_{w}$ is of size at least $\left.s\right)$. Expose all edges between $L\left(w_{i}\right)$ and $S_{w}$, and assign uniformly at random colors to all the obtained edges. Let $x \in S_{w}$ be a vertex which is connected to all the vertices in $L\left(w_{i}\right)$ and such that all the colors assigned to edges $\left\{v x \mid v \in L\left(w_{i}\right)\right\}$ are distinct and belong to $\mathcal{C}$. The existence of such a vertex follows from Claim 5.3 below. We extend $f$ by defining $f\left(w_{i}\right):=x$, update $U_{v}:=U_{v} \backslash S_{w}$ for all $v \in L\left(w_{i}\right), V^{\prime}:=V^{\prime} \backslash\{x\}$ and

$$
\mathcal{C}:=\mathcal{C} \backslash\left\{\operatorname{col} \in \mathcal{C} \mid \exists v \in L\left(w_{i}\right) \text { such that } v x \text { is colored in col }\right\} .
$$

The following two claims show that w.h.p. we manage to find the desired embedding in Phase I.

Claim 5.2 Throughout Phase I we have that $\left|A_{w}\right| \geq\left\lceil\alpha n /(4 \Delta \log n)^{2}\right\rceil$ for every vertex $w \in$ $V(H)$ which has not been embedded. 
Proof The proof of the claim is obtained from the following four observations. First, note that at the beginning of Phase I we have that $U_{v}=V(G) \backslash\{v\}$ for each $v \in V(G)$. Second, we update $U_{v}$ only after embedding a vertex $w$ for which $v \in L(w)$ (and then we delete the set $S_{w}$ which is of size $s=\left\lceil\alpha n /(4 \Delta \log n)^{2}\right\rceil$ from $\left.U_{v}\right)$. Third, every vertex $v$ is a member of at most $\Delta$ sets $L(w)$ (recall that $\Delta(H) \leq \Delta)$. Fourth, note that $\left|V^{\prime}\right| \geq\left\lceil\alpha n /\left(5 \log ^{2} n\right)\right\rceil$ throughout Phase I (recall that we do not embed $W$ in this phase).

Therefore, it follows that at any point during Phase I we have

$$
\left|U_{v} \cap V^{\prime}\right| \geq\left|V^{\prime}\right|-1-\Delta \cdot\left\lceil\frac{\alpha n}{(4 \Delta \log n)^{2}}\right\rceil,
$$

for each vertex $v \in V(G)$. Since $|L(w)| \leq \Delta$, we conclude that

$$
\left|A_{w}\right|=\left|V^{\prime} \cap\left(\cap_{v \in L(w)} U_{v}\right)\right| \geq\left|V^{\prime}\right|-\Delta-\Delta^{2}\left\lceil\frac{\alpha n}{(4 \Delta \log n)^{2}}\right\rceil \geq s,
$$

for $n$ large enough.

The next claim states that whenever we wish to embed a vertex $w$, it has at least one candidate in $V^{\prime}$.

Claim 5.3 Let $w \in V(H) \backslash W$. At the moment we try to embed $w$ there exists with probability $1-o(1 / n)$ a vertex $x \in S_{w}$ for which the following holds:

(i) $x$ is connected to all the vertices in $L(w)$, and

(ii) all the colors assigned to the edges $\{\{v, x\}: v \in L(w)\}$ are distinct and belong to $\mathcal{C}$.

Proof Let

$$
X:=\left|\left\{v \in S_{w}\left|L(w) \subseteq N_{G_{1}}(v)\right|\right\}\right| .
$$

Note that $X$ is the sum of i.i.d. indicator random variables $X_{v}$ (for all $v \in S_{w}$ ) for which $X_{v}=1$ iff $L(w) \subseteq N_{G_{1}}(v)$. Clearly, we have that (recall that $|L(w)| \leq d$ )

$$
\mathbb{E}[X] \geq s q^{d} \geq \frac{\alpha n}{(4 \Delta \log n)^{2}} \cdot \Omega\left(\frac{\log ^{5} n}{n}\right)=\Omega\left(\log ^{3} n\right) .
$$

Applying Chernoff's bound we obtain that

$$
\operatorname{Pr}[X \leq \mathbb{E}[X] / 2]=e^{-\Omega\left(\log ^{3} n\right)}=o(1 / n) .
$$

Now, note that $|\mathcal{C}| \geq \alpha|E(H)|$ during Phase I. Thus, the probability that for a vertex $x \in S_{w}$ with $L(w) \subseteq N_{G_{1}}(x)$, all the edges to $L(w)$ have different colors from $\mathcal{C}$ is at least

$$
\frac{\left(\begin{array}{l}
\mathcal{C} \\
\ell
\end{array}\right)}{((1+\alpha)|E(H)|)^{\ell}} \geq\left(\frac{\alpha|E(H)|}{(1+\alpha)|E(H)| \ell}\right)^{\ell} \geq\left(\frac{\alpha}{(1+\alpha) d}\right)^{d}=: \gamma>0
$$


where $|L(w)|=\ell$. Therefore, if $X \geq \mathbb{E}[X] / 2$ then the probability that there is no such $x$ is at most

$$
(1-\gamma)^{|X|} \leq e^{-\gamma|X|}=e^{-\Omega\left(\log ^{3} n\right)}=o(1 / n)
$$

Note that since we embed at most $n$ vertices, applying the union bound we obtain that for every vertex $w_{i}$ there exists a "good" vertex $x \in S_{w}$. Now, if $W$ is as in (1) (that is, all the vertices in $W$ are isolated in $H$ ), then we are done. Otherwise, we continue to Phase II.

Phase II: Let $V^{*}:=V(G) \backslash f(V(H) \backslash W)$. Our goal is to extend $f$ with a valid embedding of $W$ into $V^{*}$, using edges of $G_{2}$, in such a way that the resulting embedding is rainbow.

For $w \in W$ let $L(w):=f\left(N_{H}(w)\right)$ and let $\mathcal{L}=\{L(w) \mid w \in W\}$. Recall that $W$ is 2-independent and thus all the $L(w)$ 's are disjoint. Let $F=\left(\mathcal{L} \cup V^{*}, E_{F}\right)$ with edge set

$$
E_{F}:=\left\{L v \mid L \in \mathcal{L}, v \in V^{*} \text { and } \forall_{u \in L} u v \notin E\left(G_{1}\right)\right\}
$$

be the ground graph to build a bipartite auxiliary graph $\mathcal{B}\left(\mathcal{L}, V^{*}\right)$. Edges that appeared in $G_{1}$ are excluded since we can not color them again. Note that $|\mathcal{L}|=|W|=\left|V^{*}\right|$ and that by the following very rough estimate $F$ satisfies w.h.p. the conditions of Lemma 5.1 .

Claim 5.4 It holds with high probability that $\delta(F) \geq \frac{3}{4}\left|V^{*}\right|$.

Proof For every $L \in \mathcal{L}$ and $v \in V^{*}$ the edge $L v \notin E_{F}$ if and only if there exists $u \in L$ for which $u v \in E\left(G_{1}\right)$. Since $G_{1} \sim G(n, q)$, by applying Chernoff's bound it follows that w.h.p. $\Delta\left(G_{1}\right) \leq 2 n q$. Moreover, since for every $L \in \mathcal{L}$ we have that $|L| \leq \bar{d}$, it follows that $\operatorname{deg}_{F}(L) \geq\left|V^{*}\right|-\bar{d} 2 n q>3\left|V^{*}\right| / 4$. A similar argument shows that we have $\operatorname{deg}_{F}(v) \geq 3\left|V^{*}\right| / 4$ for every $v \in V^{*}$.

In the following we describe a random process that tries to create a bipartite graph $\mathcal{B}(\mathcal{L}, W) \in$ $\mathcal{B}_{\left\lceil\log ^{2} n\right\rceil-\text { out }}^{\ell}(F)$ by exposing edges from $G_{2} \backslash G_{1}$ and randomly color them. First, let

$$
\mathcal{C}:=\{c o l \in[c] \mid \exists\{u, v\} \in E(H \backslash W) \text { s.t. }\{f(u), f(v)\} \text { has color } c o l\}
$$

and note that $|\mathcal{C}| \geq \alpha|E(H)|$. Choose an arbitrary ordering $L_{1}, \ldots, L_{|\mathcal{L}|}$ of the elements in $\mathcal{L}$. Then, in step $1 \leq i \leq|\mathcal{L}|$, set $N_{i}:=N_{F}\left(L_{i}\right)$ and create $\left\lceil\log ^{2} n\right\rceil$ edges from $L_{i}$ to vertices in $N_{i}$ as follows: as long as $\left|N_{\mathcal{B}\left(\mathcal{L}, V^{*}\right)}\left(L_{i}\right)\right|<\left\lceil\log ^{2} n\right\rceil$, iteratively pick a vertex $v \in N_{i}$ uniformly at random, set $N_{i}:=N_{i} \backslash\{v\}$ and expose all edges from $v$ to vertices in $L_{i}$ and color them uniformly at random with colors from $[c]$ (note that here the process can fail if at some point $N_{i}=\emptyset$ while $\left.\left|N_{\mathcal{B}\left(\mathcal{L}, V^{*}\right)}\left(L_{i}\right)\right|<\left\lceil\log ^{2} n\right\rceil\right)$. If all the edges are contained in $G_{2}$ and if they have distinct colors that are all from the set of available colors $\mathcal{C}$, add $L_{i} v$ to $\mathcal{B}\left(\mathcal{L}, V^{*}\right)$. At the end of step $i$ remove all the colors used at edges incident to $L_{i}$,

$$
\mathcal{C}:=\mathcal{C} \backslash\left\{\operatorname{col} \in[c] \mid \exists u \in L_{i}, \exists v \in N_{\mathcal{B}\left(\mathcal{L}, V^{*}\right)}\left(L_{i}\right) \text { s.t. } u v \text { has color col }\right\}
$$


If the process succeeds then every matching $M$ in $\mathcal{B}\left(\mathcal{L}, V^{*}\right)$ is clearly rainbow in the sense that all edges in

$$
\{u v \mid \exists L v \in M \text { s.t. } u \in L\}
$$

have distinct colors that have not been used in the embedding in Phase I. It follows from Claim 5.5 below and Lemma 5.1 that the process succeeds w.h.p. and that the constructed $\mathcal{B}\left(\mathcal{L}, V^{*}\right)$ contains a perfect matching. Finally, such a perfect matching in $\mathcal{B}\left(\mathcal{L}, V^{*}\right)$ extends $f$ into a rainbow embedding of $H$ in $G$. The following claim therefore completes the proof of Theorem 1.4.

Claim 5.5 The random process that creates $\mathcal{B}\left(\mathcal{L}, V^{*}\right)$ in Phase II succeeds w.h.p. and it samples uniformly at random from $\mathcal{B}_{\left\lceil\log ^{2} n\right\rceil-\text { out }}^{\ell}(F)$.

Proof Note first that the process can only fail if in some round $1 \leq i \leq|\mathcal{L}|$ we have that $N_{i}=\emptyset$ and $\left|N_{\mathcal{B}\left(\mathcal{L}, V^{*}\right)}\left(L_{i}\right)\right|<\left\lceil\log ^{2} n\right\rceil$. It therefore suffices to show that in a fixed step $1 \leq i \leq|\mathcal{L}|$ the process creates with probability $1-o(1 / n)$ the $\left\lceil\log ^{2} n\right\rceil$ required edges. Let

$$
X_{i}:=\left\{v \in N_{F}\left(L_{i}\right)\left|L_{i} \subseteq N_{G_{2}}(v)\right|\right\} .
$$

Note that $\left|X_{i}\right|$ is the sum of i.i.d. indicator random variables $X_{i, v}$ (for all $v \in N_{F}\left(L_{i}\right)$ ) for which $X_{i, v}=1$ iff $L_{i} \subseteq N_{G_{2}}(v)$. Clearly, we have that (recall that $\left|L_{i}\right| \leq \bar{d} \leq d$ )

$$
\mathbb{E}\left[\left|X_{i}\right|\right] \geq\left|N_{F}\left(L_{i}\right)\right| q^{d} \geq \delta(F) \cdot \Omega\left(\frac{\log ^{5} n}{n}\right) \geq \frac{3}{4} \cdot \frac{\alpha n}{5 \log ^{2} n} \cdot \Omega\left(\frac{\log ^{5} n}{n}\right)=\Omega\left(\log ^{3} n\right) .
$$

Applying Chernoff's bound we obtain that

$$
\operatorname{Pr}\left[\left|X_{i}\right| \leq \mathbb{E}\left[\left|X_{i}\right|\right] / 2\right]=e^{-\Omega\left(\log ^{3} n\right)}=o(1 / n) .
$$

Next, let

$$
Y_{i}:=\left\{v \in X_{i} \mid \text { all edges in } E\left(L_{i}, v\right) \text { have distinct colors from } \mathcal{C}\right\} .
$$

Note that $\left|Y_{i}\right|$ is the sum of i.i.d. indicator variables $Y_{i, v}$ (for all $v \in X_{i}$ ) for which $Y_{i, v}=1$ iff all edges in $E\left(L_{i}, v\right)$ have distinct colors from $\mathcal{C}$. Since we have by (4) that $|E(W, V \backslash W)| \leq$ $\alpha|E(H)| /\left(2\left\lceil\log ^{2} n\right\rceil\right)$ and we remove for each edge in $E(W, V \backslash W)$ at most $\left\lceil\log ^{2} n\right\rceil$ colors from $\mathcal{C}$, the number of available colors in $\mathcal{C}$ is always at least $\alpha|E(H)| / 2$. Thus, the probability that for a vertex $v \in X_{i}$ all the edges to $L_{i}$ have different colors from $\mathcal{C}$ is at least

$$
p_{i}=\frac{\left(\begin{array}{l}
\mathcal{C} \\
\ell
\end{array}\right)}{((1+\alpha)|E(H)|)^{\ell}} \geq\left(\frac{\alpha|E(H)| / 2}{(1+\alpha)|E(H)| \ell}\right)^{\ell} \geq\left(\frac{\alpha}{(1+\alpha) 2 \bar{d}}\right)^{\bar{d}}=: \gamma>0,
$$

where $\left|L_{i}\right|=\ell \leq \bar{d}$, and this lower bound for $p_{i}$ holds independently of all other color assignments in previous steps. Therefore, if $\left|X_{i}\right| \geq \mathbb{E}\left[\left|X_{i}\right|\right] / 2$, then the expectation of $\left|Y_{i}\right|$ is at least

$$
\mathbb{E}\left[\left|Y_{i}\right|\right] \geq\left|X_{i}\right| \cdot \gamma=\Omega\left(\mathbb{E}\left[\left|X_{i}\right|\right]\right)=\Omega\left(\log ^{3} n\right)
$$


and it follows from Chernoff's bound that

$$
\operatorname{Pr}\left[\left|Y_{i}\right|<\frac{\mathbb{E}\left[\left|Y_{i}\right|\right]}{2}|| X_{i} \mid \geq \frac{\mathbb{E}\left[\left|X_{i}\right|\right]}{2}\right]=e^{-\Omega\left(\log ^{3} n\right)}=o(1 / n) .
$$

Combining (5) and (6) we conclude that the probability that our process fails is at most

$$
\sum_{i=1}^{|\mathcal{L}|} \operatorname{Pr}\left[\left|Y_{i}\right| \leq\left\lceil\log ^{2} n\right\rceil\right] \leq|\mathcal{L}| \cdot o(1 / n)=o(1)
$$

Finally, since we choose a random ordering of the neighbors of $L_{i}$, every $\left\lceil\log ^{2} n\right\rceil$-tuple of neighbors of $L_{i}$ has the same probability to be part of $\mathcal{B}\left(\mathcal{L}, V^{*}\right)$ and the process therefore samples an element of $\mathcal{B}_{\left\lceil\log ^{2} n\right\rceil-\text { out }}^{\ell}(F)$ uniformly at random.

Acknowledgment. The first author is grateful to Michael Krivelevich for pointing out the problem of finding a rainbow embedding in random graphs, and to Benny Sudakov for many helpful and valuable conversations. The authors are also grateful to Peter Allen for giving some useful comments on an earlier draft. Last but not least, the authors are grateful to the anonymous referees for many valuable comments.

\section{References}

[1] Peter Allen, Julia Böttcher, Hiep Hàn, Yoshiharu Kohayakawa, and Yury Person, Sparse blow-up lemmas, preprint.

[2] Noga Alon and Vera Asodi, Sparse universal graphs, Journal of Computational and Applied Mathematics 142 (2002), no. 1, 1-11.

[3] Noga Alon and Michael Capalbo, Sparse universal graphs for bounded-degree graphs, Random Structures \& Algorithms 31 (2007), no. 2, 123-133.

[4] Noga Alon, Michael Capalbo, Yoshiharu Kohayakawa, Vojtech Rodl, Andrzej Rucinski, and Endre Szemerédi, Universality and tolerance, Proceedings of the 41st IEEE Symposium on Foundations of Computer Science, IEEE, 2000, pp. 14-21.

[5] Noga Alon and Zoltán Füredi, Spanning subgraphs of random graphs, Graphs and Combinatorics 8 (1992), no. 1, 91-94.

[6] Noga Alon, Michael Krivelevich, and Benny Sudakov, Embedding nearly-spanning bounded degree trees, Combinatorica 27 (2007), no. 6, 629-644.

[7] Laszlo Babai, Fan R. K. Chung, Paul Erdős, Ronald L. Graham, and Joel Spencer, On graphs which contain all sparse graphs, Ann. Discrete Math 12 (1982), 21-26. 
[8] József Balogh, Béla Csaba, Martin Pei, and Wojciech Samotij, Large bounded degree trees in expanding graphs, Electronic Journal of Combinatorics 17 (2010), no. 1, R6.

[9] József Balogh, Béla Csaba, and Wojciech Samotij, Local resilience of almost spanning trees in random graphs, Random Structures \& Algorithms 38 (2011), no. 1-2, 121-139.

[10] Sandeep N. Bhatt, Fan R. K. Chung, Frank T. Leighton, and Arnold L. Rosenberg, Universal graphs for bounded-degree trees and planar graphs, SIAM Journal on Discrete Mathematics 2 (1989), no. 2, 145-155.

[11] Julia Böttcher, Klaas P. Pruessmann, Anusch Taraz, and Andreas Würfl, Bandwidth, expansion, treewidth, separators and universality for bounded-degree graphs, European Journal of Combinatorics 31 (2010), no. 5, 1217-1227.

[12] Michael Capalbo, Explicit sparse almost-universal graphs for $\mathcal{G}\left(n, \frac{k}{n}\right)$, Random Structures \& Algorithms 37 (2010), no. 4, 437-454.

[13] Fan R. K. Chung and Ronald L. Graham, On universal graphs for spanning trees, Journal of the London Mathematical Society 2 (1983), no. 2, 203-211.

[14] Colin Cooper and Alan Frieze, Multi-coloured hamilton cycles in random edge-coloured graphs, Combinatorics Probability and Computing 11 (2002), no. 2, 129-133.

[15] Domingos Dellamonica Jr, Yoshiharu Kohayakawa, Vojtěch Rödl, and Andrzej Ruciński, An improved upper bound on the density of universal random graphs, LATIN 2012: Theoretical Informatics, Springer, 2012, pp. 231-242.

[16] Devdatt P. Dubhashi and Alessandro Panconesi, Concentration of measure for the analysis of randomized algorithms, Cambridge University Press, 2009.

[17] Alan Frieze and Po-Shen Loh, Rainbow hamilton cycles in random graphs, Random Structures \& Algorithms 44 (2014), no. 3, 328-354.

[18] Svante Janson, Tomasz Luczak, and Andrzej Rucinski, Random graphs, vol. 45, John Wiley \& Sons, 2011.

[19] Daniel Johannsen, Michael Krivelevich, and Wojciech Samotij, Expanders are universal for the class of all spanning trees, Combinatorics, Probability and Computing 22 (2013), no. $02,253-281$.

[20] Anders Johansson, Jeff Kahn, and Van Vu, Factors in random graphs, Random Structures \& Algorithms 33 (2008), no. 1, 1-28.

[21] Jeong Han Kim and Sang June Lee, Universality of random graphs for graphs of maximum degree two, arXiv preprint arXiv:1310.5873 (2013).

[22] Andrzej Ruciński, Matching and covering the vertices of a random graph by copies of a given graph, Discrete mathematics 105 (1992), no. 1, 185-197. 
[23] Douglas B. West, Introduction to graph theory, vol. 2, Prentice hall Upper Saddle River, 2001. 